OPEN ACCESS

Edited by:

Elizabeth Chrastil,

University of California,

Santa Barbara, United States

Reviewed by:

Sarah H. Creem-Regehr.

The University of Utah, United States

Jon Kelly,

lowa State University, United States

*Correspondence:

Michael J. Starrett

mistarrett@gmail.com

Received: 29 March 2018

Accepted: 22 June 2018

Published: 11 July 2018

Citation:

Starrett MJ and Ekstrom AD (2018)

Perspective: Assessing the Flexible

Acquisition, Integration, and Deployment of Human Spatial Representations and Information.

Front. Hum. Neurosci. 12:281. doi: 10.3389/fnhum.2018.00281

\section{Perspective: Assessing the Flexible Acquisition, Integration, and Deployment of Human Spatial Representations and Information}

\author{
Michael J. Starrett ${ }^{1,2,3 *}$ and Arne D. Ekstrom ${ }^{1,2,3,4}$ \\ 'Department of Psychology, University of Arizona, Tucson, AZ, United States, ${ }^{2}$ Department of Psychology, University \\ of California, Davis, Davis, CA, United States, ${ }^{3}$ Center for Neuroscience, University of California, Davis, Davis, CA, \\ United States, ${ }^{4}$ Neuroscience Graduate Group, University of California, Davis, Davis, CA, United States
}

Studying human spatial navigation in the lab can be challenging, particularly when including non-invasive neural measures like functional magnetic resonance imaging (fMRI) and scalp encephalography (EEG). While there is broad consensus that human spatial navigation involves both egocentric (self-referenced) and allocentric (worldreferenced) coding schemes, exactly how these can be measured in ecologically meaningful situations remains controversial. Here, we explore these two forms of representation and how we might better measure them by reviewing commonly used spatial memory tasks and proposing a new task: the relative vector discrimination (RVD) task. Additionally, we explore how different encoding modalities (desktop virtual reality, immersive virtual reality, maps, and real-world navigation) might alter how egocentric and allocentric representations manifest. Specifically, we discuss desktop virtual reality vs. more immersive forms of navigation that better approximate realworld situations, and the extent to which less immersive encoding modalities alter neural and cognitive codes engaged during navigation more generally. We conclude that while encoding modality likely alters navigation-related codes to some degree, including egocentric and allocentric representations, it does not fundamentally change the underlying representations. Considering these arguments together, we suggest that tools to study human navigation in the lab, such as desktop virtual reality, provide overall a reasonable approximation of in vivo navigation, with some caveats.

Keywords: spatial representations, spatial information, navigation, egocentric, allocentric, virtual reality, relative vector discrimination (RVD) task

\section{INTRODUCTION}

Cognitive neuroscience provides a wide variety of behavioral and neural tools to assay cognitive processes and neural systems that underlie human spatial navigation. However, like any measurement tool in science, there are limitations to how they can be applied and exactly what information they provide. In terms of behavioral measures for spatial knowledge, two pointing tasks have been widely used: the scene and orientation-dependent pointing (SOP) task and the judgments of relative direction (JRD) pointing task. In the SOP task, shown in Figure 1A, participants are oriented within the environment and then asked to point to target objects. In the VR version, all targets are removed, and background information provides visual orienting 
information (e.g., Zhang et al., 2014). In the real-world version, participants are blindfolded but oriented within the environment via body-based input (e.g., Wang and Spelke, 2000). Conversely, the JRD task is conducted with participants either disoriented within the environment, or moved to a different environment, where they are provided a triad of targets (delivered via text or by the experimenter, verbally; "Imagine standing at A, facing B; point to C."). The first two targets serve to established an imagined heading to orient to while participants point to the third target in the triad (Shelton and McNamara, 2001; Mou et al., 2004; Waller and Hodgson, 2006; Starrett et al., in press). An example trial is shown in Figure 1B. There are systematic differences in what is measured by each task, and both tasks have limitations related to exactly what they measure in terms of underlying spatial representations (Mou et al., 2004; Kelly et al., 2007; Ekstrom et al., 2014; Zhang et al., 2014).

In terms of neural assays, functional magnetic resonance imaging (fMRI) requires participants to lay supine while navigating in virtual reality (VR), and challenges remain for conducting scalp EEG during real-world exploration (but see Gramann et al., 2010). Our main focus in this perspective is therefore to consider the limitations imposed by the SOP and JRD tasks and studying navigation in VR more generally. We also consider the valuable information we can nonetheless glean from them in terms of how we navigate and suggest a new relative vector discrimination (RVD) task (see Figure 1) aimed to better describe spatial memory for allocentric reference frames and the flexibility of representations across various spatial task demands.

One way to consider the relative demands of the SOP and JRD tasks is along an egocentric to allocentric continuum (see Figure 2), which also allows us to consider how different levels of immersion in VR might affect where they fall on this spectrum. We suggest that future experiments should focus on how spatial information manifests and is accumulated during various encoding modalities (e.g., route versus map learning). Separately, we consider how this information might be strategically deployed depending on flexible task demands during retrieval (e.g., SOP, JRD, map drawing, etc.). We can potentially better model and understand the nature of representations underlying human spatial navigation by considering how spatial information is first encoded along the egocentric to allocentric continuum, and then subsequently retrieved depending on the task demands.

\section{THE EGOCENTRIC TO ALLOCENTRIC SPECTRUM: WHERE DO THE DIFFERENT POINTING TASKS SIT?}

One of the most widely used distinctions in spatial navigation involves the idea of two fundamentally different forms of representations, egocentric vs. allocentric. Briefly, navigation involving an egocentric representation employs a coordinate system referenced to one's current position and facing direction and is most familiar in navigating our immediate, peripersonal space. Examples include knowing where a chair is in front of us so that we can avoid colliding with it when we get up or reaching for a mug next to us. One disadvantage of egocentric coordinates, however, is they change constantly as the navigator moves, requiring continuous updating of one's position. In contrast, an allocentric representation involves reference to objects that remain constant in the external world. For example, using other landmarks to remember how to get to a goal. A disadvantage of an allocentric representation is that it requires reference, and memory for, multiple landmarks.

There is at least some evidence that the SOP task assays primarily egocentric forms of spatial representation while the JRD task assays primarily allocentric forms of representation (Figure 2). In particular, there is general agreement that the SOP task is primarily egocentric, provided that participants are oriented when pointing and that the dependent measure is absolute pointing error (Wang and Spelke, 2000; Holmes and Sholl, 2005; Waller and Hodgson, 2006; Zhang et al., 2014). In contrast, there is significantly less agreement regarding the JRD task and the extent to which it provides primarily allocentric, or some complex combination of egocentric and allocentric, information (Ekstrom et al., 2017). Specifically, given that imagined, first-person headings are a fundamental part of the task ("imagine you are facing X"), it seems difficult to fully discount the contributions of egocentric viewpoint information from JRDs (Ekstrom et al., 2014). Indeed, previous work has demonstrated a bias to perform better when pointing at targets in the forward hemifield of the imagined heading and that this bias is weaker or absent when information is learned from a map (Sholl, 1999; Kelly and McNamara, 2009), suggesting that spatial information is either acquired or deployed differently across learning modality even for the same retrieval task. In addition, a recent article by Wang (2017) demonstrated that tasks like the Morris Water Maze, often argued to rely on allocentric representations (Morris et al., 1986), can also be solved using egocentric coordinates (Wang, 2017). Finally, the JRD task does not involve an estimate of distance, an important component of allocentric representation more generally (because egocentric representations are more likely to involve viewpoint and bearingdependent "snapshots," distance is likely less relevant).

\section{THE RELATIVE VECTOR DISCRIMINATION (RVD) TASK: A MORE ALLOCENTRIC "ALLOCENTRIC" TASK}

We propose a new RVD spatial memory task to provide a fundamentally more (although still not purely) allocentric task than the JRD task and thus a better foil for the SOP task (see Figure 1). Inspired by the task used by Han and Becker (2014), the RVD task is framed in the third-person, with participants receiving a top-down view of the locations of two target stores and placing a third freely (Figure 1C). In the RVD task, participants are presented with a blank screen showing two "anchor" landmarks positioned relative to one another on the screen. The position of these landmarks on the screen is fixed. Depending on the vector defined by the positions of the anchor landmarks, participants will be required to place a third target landmark on the screen relative to the anchor landmarks (Figure 1C). One benefit of the RVD task is that it provides a 
A

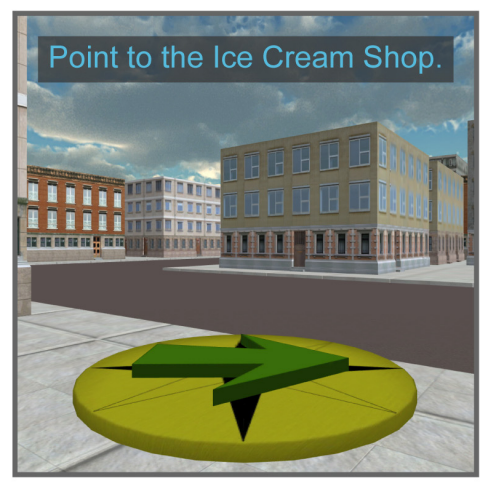

B

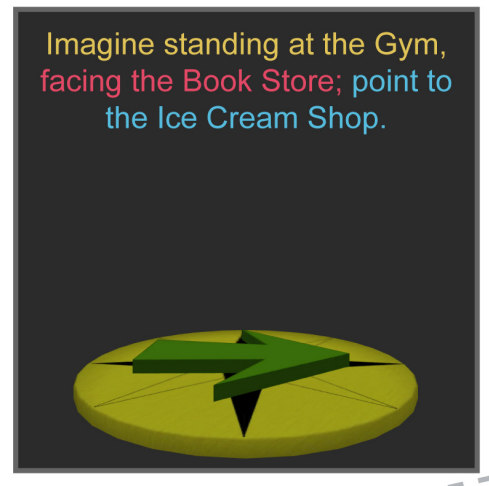

Imagine standing at the Gym,

the Ice Cream Shop.

D

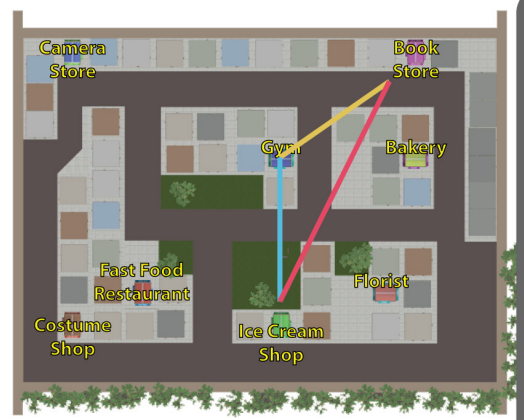

E

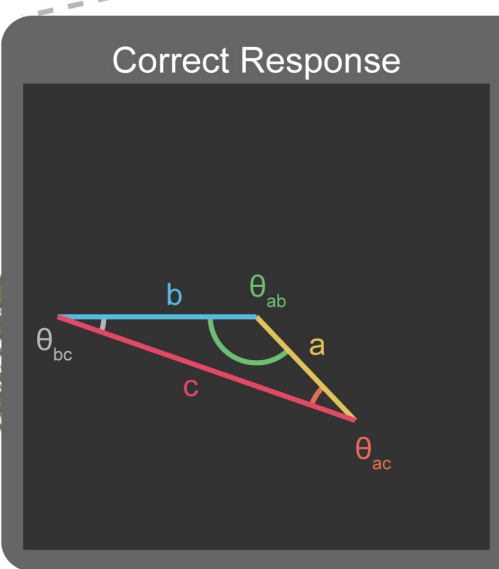

C
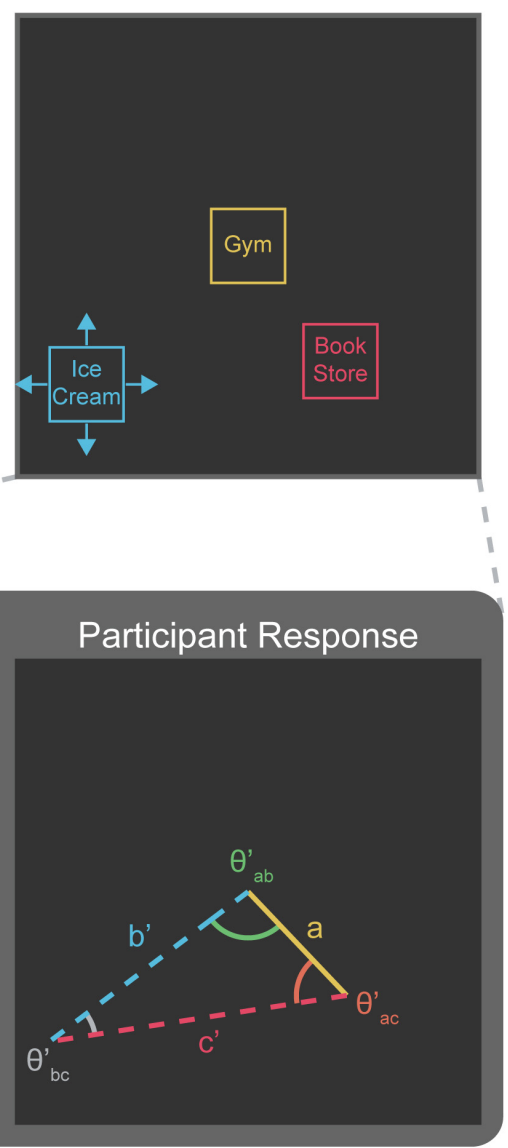

$\mathbf{F}$

Allocentric

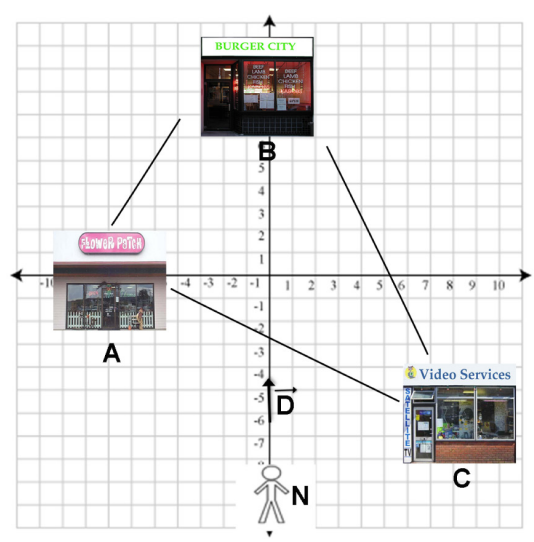

Egocentric

2.

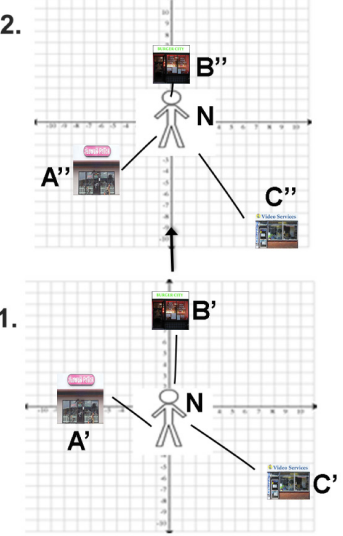

FIGURE 1 | Pointing tasks. Single-trial examples for computer versions of the scene and orientation-dependent (SOP) pointing task (A), the judgments of relative direction (JRD) pointing task (B), and the proposed relative vector discrimination (RVD) task (C). In each example, participants' memory for the location of the lce Cream Shop (blue text) is being tested. Example trials are based on the virtual environment used by Starrett et al. (in press) (D). Unlike the JRD and SOP tasks, which only yield angular precision estimates, the RVD task yields both angular and distance information [note that the anchor vector (yellow line) is common across the correct and participant response, and the placement of the target store establishes the remaining two legs of a triangle (red and blue lines)] (E). A reproduction of Figure 1 from Ekstrom et al. (2017) shows the cartesian relationship between allocentric (left panel) and egocentric (right panel) as a displacement vector from the navigator (F). 


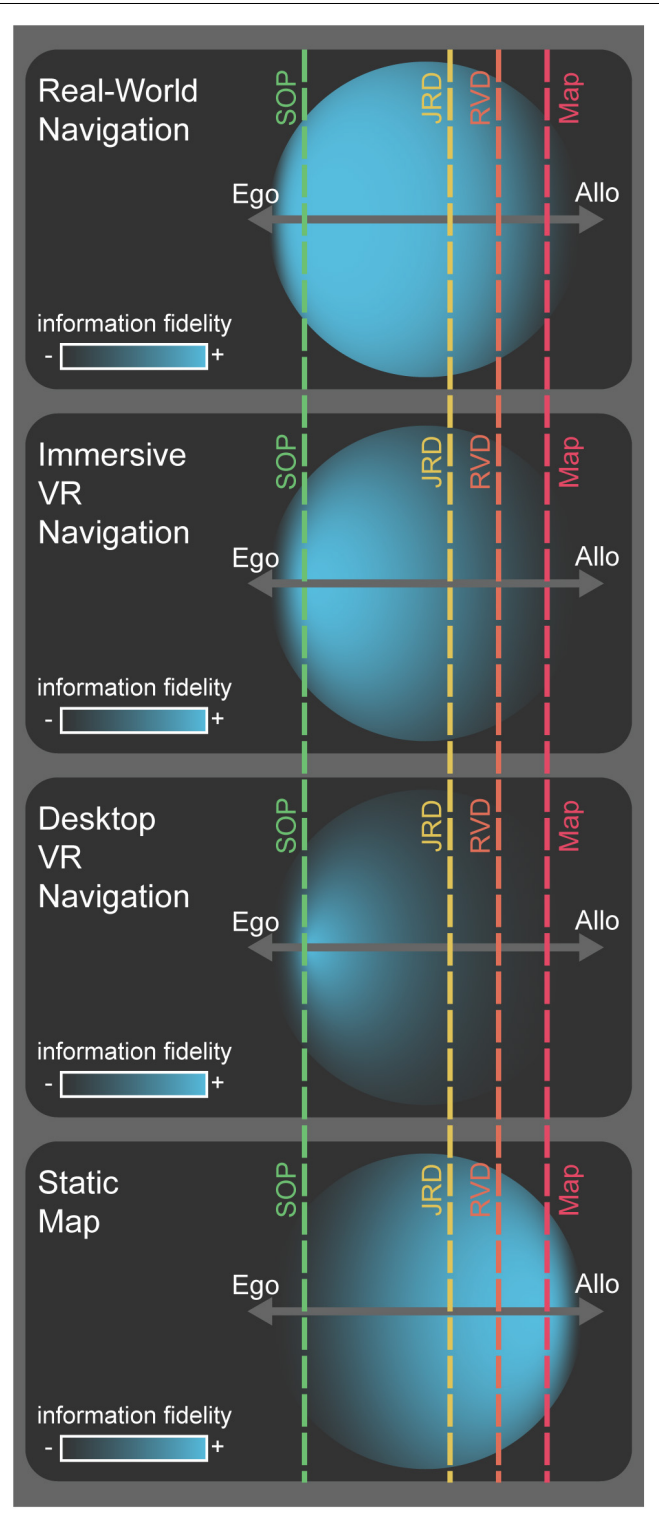

FIGURE 2 | Conceptual model for the encoding and deployment of spatial information. Hypothetical spatial information (blue circles) acquired during early encoding of a novel environment via various encoding modalities (labels, left). Each example shows the fidelity of spatial information acquired (density and center of mass for blue circles) along an egocentric-to-allocentric continuum (dark gray, horizontal axes). The extent to which the circles project outward from the egocentric-allocentric axis illustrates information that, while not strictly spatial in nature (e.g., semantic or episodic memory), can be used to derive spatial information or drive inferences about the environment. The offset of the vertical, dashed, lines from the center of the egocentricallocentric axis represents the hypothetical proportion to which that task depends more on egocentric or allocentric reference frames for its optimal solution (SOP: green, JRD: yellow, RVD: orange, map drawing: red).

measure of both angle and distance, as well as latency, within the same task. Error can be quantified by comparing the geometry of the triangle created by the anchor vector and the correct placement vectors with the anchor vector and the participant's placement vectors (Figure 1E).
While the RVD task differs in several important ways from the SOP and JRD, performance on all three tasks is dependent on similar spatial memory principles such as environmental geometry, salient landmarks, and learned viewpoints or routes (depending on learning modality). For example, the anchor vector in the RVD task can be thought of as an analog to the imagined heading in JRD task or the oriented perspective in the SOP task. Therefore, the same types of independent manipulations can be applied to the RVD task (e.g., alignment with learned perspectives or environmental geometry). In fact, as suggested by the title of this section, the primary objective in creating this task is to yield a dependent variable comparable to that of the JRD, but with intrinsically more allocentric demands based on how the task is framed. This is illustrated in Figures 1A-E, where the orientation, imagined heading, and anchor vector in the SOP, JRD, and RVD, respectively are identical. The linearly transformable relationship between egocentric and allocentric cartesian coordinates is also illustrated in Figure 1 of Ekstrom et al. (2017), shown in Figure 1F. Therefore, if these tasks were identical, the triangles derived from responses on any of them should theoretically be geometrically similar (i.e., the corresponding angles should be equal), if not identical. Any deformation would be indicative of differences in task demands. Notably, with the inclusion of distance estimates in the RVD, other metrics comparing the deformation of entire shapes may be used to compare triangles derived from participants' responses against those derived from the correct response, such as those put forward by Basri et al. (1998).

When implementing the RVD task, there are several important parameters and aspects of the task to consider. (1) The degree of potential egocentricity can be manipulated even within the parameters of the RVD task (further supporting the idea of flexible spatial demands for retrieval tasks). For example, the anchor vector could be constrained to always originate from the center of the screen (as in the example trial shown in Figure 1C). While this may prove useful for experimental designs that require fixation, such as fMRI or eye tracking paradigms, doing so also limits the extent to which experimenters can rule out participants' reliance on a central "self-" centered anchor within their visual field. Other parameters that may affect the egocentric versus allocentric demands of the RVD task include length of the anchor vector relative to a learned viewpoint (particularly for map learning), the orientation of the anchor vector relative to learned viewpoint (global map orientation for map learning or possibly initial or final viewpoints during navigation), and potentially others. (2) Whether RVD performance is being compared with performance on other spatial memory tasks such as the SOP, JRD, or map drawing. In the case of the SOP and JRD tasks, which traditionally do not include distance estimates, it may prove beneficial to constrain the anchor vector to originate from the origin (as in Figure 1C) to provide a clear comparison angle and analog for imagined heading used in the SOP and JRD. Additionally, the length of the anchor vector could be constrained either to be identical on every trial or scaled to corresponding lengths relative to the geometry of the environment if the experimenter wishes to attempt to control for angular biases related to distance. Ultimately, the 
consideration of these parameters will depend on the design of the experiment and the questions being addressed (Waller and Hodgson, 2013).

The RVD task thus provides an additional position along the egocentric to allocentric continuum of spatial information (see Figure 2) that can be probed during recall. This will allow future experiments to expand on previous findings comparing the JRD and SOP tasks (Waller and Hodgson, 2006; Zhang et al., 2014) by observing and contrasting performance on the RVD task across encoding modalities (routes/maps) relative to the JRD and SOP tasks. We hypothesize that the RVD task can be used to coerce the deployment of more allocentric spatial information in well-learned environments or the conversion of egocentric information to make inferences from an imagined or low-fidelity allocentric reference frame (Figure 2), consistent with previous suggestions regarding how participants often utilize allocentric representations (Mou et al., 2006; Newman et al., 2007).

\section{THE SPACE BETWEEN REFERENCE FRAMES: ENCODING MODALITIES, RETRIEVAL DEMANDS, AND HOW THEY INTERACT WITH SPATIAL KNOWLEDGE}

As suggested by the BBB model (Byrne et al., 2007) but worked out in detail in a recent computational paper (Wang, 2017), the primary difference between egocentric and allocentric representations involves keeping track of one's displacement (Figure 1F; reproduction of Figure 1 from Ekstrom et al., 2017) (see also Ekstrom and Isham, 2017, Figure 1; Wang, 2017, Figure 2). As described earlier, in an egocentric reference frame, the coordinates for one's position stay constant while those for landmarks continuously change. In contrast, in an allocentric reference frame, the positions of landmarks stay constant while those of the self continuously change. Thus, the allocentric reference seems computationally more efficient because only the movement of the navigator needs to be maintained, and thus eventual conversion of egocentric to primarily allocentric coordinates would appear advantageous. Consistent with this idea, during navigation of well-known environments, participants appear to prefer allocentric reference frames, but when allocentric information is not reliable or is of low fidelity, egocentric reference frames dominate (Mou et al., 2006; Newman et al., 2007).

While it is possible to define and distinguish egocentric and allocentric reference frames mathematically and anecdotally, and why an allocentric reference in particular might be most advantageous for navigating, in practice, the interaction and dynamic use of either or both can be difficult to parse, particularly given that the main difference involves a simple linear transformation (adding/subtracting one's displacement). Consider the example of driving with a global positioning system (GPS). In this scenario, it is unlikely that the GPS or the driver's view of the road will be used in isolation. Most likely, attention will constantly shift from the road to the GPS and back, all the while updating and integrating information from each source.
This example illustrates how navigation in the modern world rarely involves a static egocentric or allocentric reference frame.

Moreover, the specific reference frames used may not be purely egocentric or allocentric. While GPS devices do show a map view of the environment, this map is often updated such that an icon indicating the user's current position is constantly centered and sometimes even facing the current direction of travel, introducing an egocentric element. The GPS represents an example of a hybrid reference frame that may be integrated with, translated to, or even represented independently from more egocentric and allocentric reference frames as one navigates (Trullier et al., 1997; Eichenbaum, 2017). Such hybrid information could facilitate more rapid integration with realworld egocentric information in lieu of actual topological or survey knowledge by placing the onus of any computational conversions or representations on the GPS rather than areas proposed to be important for egocentric to allocentric conversion like retrosplenial cortex (Burgess, 2006; Byrne et al., 2007; Epstein, 2008; Ekstrom et al., 2017).

In Figure 2, we present a conceptual model for describing spatial information along the egocentric-to-allocentric continuum. Spatial information is indicated by the blue area of the circle for which density denotes more high-fidelity information and the center of mass shows the utility of that information for task demands ranging from egocentric to allocentric. The position, density, and dispersion of the spatial information circles are largely influenced by learning modality (illustrated by contrasts between panels in Figure 2), but for both egocentric and allocentric information, participants acquire varying amounts and fidelity in each learning condition. Various retrieval task demands are depicted by vertical, dashed lines, with the offset from the center of the continuum illustrating the relative egocentric-allocentric dependence of the task. As emphasized in the figure, not only are the learning modality and retrieval task demands critical individually, but so is the interaction between two. Here, spatial information is represented by a 2-dimensional circle along a 1-dimensional egocentricallocentric axis. The extent to which the circle projects outward, orthogonally, from the egocentric-allocentric axis is intended to represent memory or knowledge that may not be specific to the environment being learned, like semantic (many cities are arranged in blocks or grids) or episodic memory (a car almost hit me when I crossed that street once). Thus, this conceptual model attempts to account for not only spatial information categorized from the "primary" reference frame, but also from the nondominant reference frame and more abstract information like Bayesian priors or heuristics.

The model illustrates several important properties of how we encode and deploy spatial information, which can be impacted by (1) the encoding modality (e.g., routes or maps), (2) the strength or fidelity of the spatial information encoded (also partially dependent on the encoding modality), (3) the optimal reference frame used for solving a specific spatial memory task (note that none of these tasks can guarantee how participants will solve a retrieval task, rather only encourage selection of a desired, optimal solution), and (4) the ability to deploy prior knowledge and heuristics to make inferences using one reference frame when 
spatial information is encoded primarily from the other reference frame.

Here, we define heuristics as "up is north" (Brunye et al., 2012) or the well-described advantage that comes with remembering facing locations aligned with the axes of a rectangle compared to misaligned (e.g., Mou and McNamara, 2002; Starrett et al., in press). In the case of using rectangles as a heuristic, memory for the location of a target can be bound to the geometry of the rectangle, in that any points defined by its orthogonal distance to each side of the rectangle share the same principle axes relative to the environment. The selection of a singular, bipolar axis or two primary axes, akin to cardinal directions on a map, is consistent with how we often learn from and interpret maps and is thus a familiar and efficient way to remember any space with rectangular properties. In terms of our model, this would involve using information in denser areas or from other spatial representations to fill in less dense areas (Figure 2), perhaps temporarily while more high-fidelity information is acquired to establish that reference frame. In this way, our model helps explain several previously described phenomena in the human spatial navigation literature that lack a clear theoretical connection with egocentric vs. allocentric representation.

\section{HOW EGOCENTRIC AND ALLOCENTRIC REPRESENTATIONS INTERACT WITH ENCODING MODALITY}

Understanding the neural basis of egocentric and allocentric representations is an important research endeavor (for recent review, see Ekstrom et al., 2017). One important limitation inherent in most neural recordings from humans is that they have traditionally been limited to desktop interfaces, which lack many of the characteristics of real-world navigation. Namely, desktop VR does not provide idiothetic, self-motion cues because participants sit stationary, and desktop VR may introduce conflict between real-world and virtual allothetic cues. Even the rendering of optic flow, while still 3-dimensional, lacks the exact stereoscopic immersion of real-world experiences (see Snow et al., 2014). Specifically, in the context of navigation as a means for encoding spatial representations, the removal of such selfbased information could fundamentally alter the neural processes and mechanisms being studied under such conditions relative to real-world (for review, see Taube et al., 2013). For example, desktop VR lacks true head-turns, resulting in little or no vestibular information during such navigation tasks. Vestibular lesions have been shown to significantly alter hippocampal theta oscillations in rats (Russell et al., 2006), an important neural signal related to spatial navigation, raising the possibility that the lack of head-direction input could fundamentally alter these codes. In humans, diminished vestibular information relative to real-world navigation may have downstream effects on other types of spatial processing neurons such as path cells, boundaryvector cells, or head-direction cells (Ekstrom, 2010; Jacobs et al., 2010; Taube et al., 2013).

To what extent does the lack of head-direction input limit the nature of spatial representations that can be assayed with desktop VR in humans? Invasive recordings of the hippocampus in humans, monkeys, and rats have all identified place cells, with Miller et al. (2013) showing that during later free recall of items associated with locations in a virtual environment, the same or nearby hippocampal place cells fired. These findings suggest that desktop VR, and even desktop presented stimuli in the absence of immersive scene information, do capture sufficient information to recapitulate neural codes from the real world. Notably, view-coding cells are also present in both monkeys and humans, suggesting a specific mechanism that could favor view-dependent, VR-based navigation (Ekstrom, 2015). Thus, the presence of place cells across modalities and species as well as view-coding mechanisms in primates, argue against the idea that the lack of explicit head-direction input during desktop VR somehow fundamentally changes how we code space during navigation.

Similarly, low-frequency, movement-related theta oscillations in the hippocampus, semi-periodic fluctuations in the local field potential that manifest during navigation, are present during desktop VR (Watrous et al., 2011, 2013; Bohbot et al., 2017), retrieval of spatial information (Ekstrom et al., 2007), encoding and retrieval of verbal associations (Sederberg et al., 2003; Yaffe et al., 2014), and during real-world navigation (Aghajan et al., 2017; Bohbot et al., 2017). One possibility is that the frequency of theta oscillations during real-world navigation in humans might be higher than VR, similar to higher frequency theta oscillations in rodents (Yassa, 2018). This in turn might seem to bolster the argument that VR and real-world navigation alter underlying neural representations (Aghajan et al., 2017). It is important to note, however, that the wireless hippocampal recordings used by Aghajan et al. (2017) could not detect oscillations below $4 \mathrm{~Hz}$ due to hardware-enforced, bandpass filtering. Indeed, Bohbot et al. (2017) used wired recordings during free ambulation and analyzed frequencies as low as $1 \mathrm{~Hz}$, finding that low frequency oscillations were present across the range of $1-12 \mathrm{~Hz}$, with only a slight difference in frequency across all electrode recordings for VR vs. real-world movements (see also Aghajan et al., 2017, Supplementary Figure 4 for examples from their wired recordings). Thus, low-frequency hippocampal theta oscillations, an important navigation-related neural signal, are present during a variety of immersed and non-immersed memory and navigation tasks to comparable extents, all of which appear sufficient to induce its presence.

If the lack of vestibular and other whole-body input did dramatically affect our underlying spatial codes, we might expect significant changes in how we learn environments with a full range of body-based cues compared to an impoverished set, such as navigating in desktop VR. Past behavioral studies have investigated these issues, with one early study suggesting that VR learning transfers only minimally to real-world environments (Kozak et al., 1993). A later study, however, by Richardson et al. (1999) only observed diminished performance when pointing tasks required participants to remember spatial relationships from different floors of a virtual building. One major issue with these early studies, however, is that VR technology was in its relative infancy and the complexity of visual displays and environmental geometry were relatively limited. With VR 
capturing real-world environments to only a limited extent, it is not surprising that transfer was minimal.

Recent experiments have begun to test learning in VR under a richer set of conditions than simply comparing desktop VR to real-world navigation, and in particular, the advent of the head-mounted display has allowed researchers to render complex visual environments as a participant freely ambulates within it. In one set of experiments participants had to search virtual birdhouses for a target. Importantly, they tested participants either while standing still and using a controller for rotations and translation, standing still but physically rotating while using a controller for only translations, or physically walking and turning. Results showed that physically walking and turning with the head-mounted display, i.e., the combination of vestibular, proprioceptive, and somatosensory information, significantly improved performance (Ruddle and Lessels, 2006, 2009) compared to the other conditions; thus, vestibular input, on its own, did not seem to be as important as the combination of multimodal cues.

More recently, Chrastil and Warren (2013) used a hedgemaze navigation task and assessed performance based on the later use of shortcuts to target locations. Participants learned the maze either by watching a video, being moved through the environment in a wheelchair, or by walking. Performance in the wheelchair condition, which provided rich vestibular information, was statistically equivalent to performance in the video condition, which was slightly above chance. In contrast, performance was better in the walking condition, which provided vestibular and proprioceptive input, than video condition. Other studies aimed at dissociating the contributions of proprioception (walking) and vestibular (turning) information have found that rotational information alone contributes minimally to performance on spatial estimates (Ruddle et al., 2011). While other studies have yielded conflicting results with regard to the importance of translational versus rotational body-based cues (see Chance et al., 1998), one common finding across these studies is that the full-range of multimodal body-based input seems to boost performance during navigation with the majority of findings suggesting that vestibular input alone is not necessary for the normal expression of spatial representations in humans. The question becomes even more complex when accounting for the plethora of ever-evolving interfaces used for immersive interaction with large-scale virtual environments, (head-mounted displays, CAVEs, omnidirectional treadmills), which introduce further nuances to the information available for encoding during spatial learning and should be considered when developing navigation and spatial learning paradigms (for a somewhat recent review, see Waller and Hodgson, 2013; see also: He et al., 2017; Paris et al., 2017; Starrett et al., in press).

While it is clear that there are some behavioral differences between learning with the full-range of body-based input vs. an impoverished set of cues, importantly, at least some of this may be attributable to memory-related effects (Tulving and Thomson, 1973). In other words, when we have a richer set of cues to encode information in the first place, we will benefit from these multiple cues during retrieval to a greater extent than when encoding and retrieval occur with a smaller number or different set of cues. A greater number of modalities also mean that different learning systems can work in parallel, providing the potential for faster learning. Thus, we believe that the evidence argues strongly against the idea that vestibular input is necessary for "normal" spatial navigation, by which we intend to say that navigation derived from visual input alone is largely sufficient for the types of modality independent spatial representations we form during navigation (see Waller and Hodgson, 2013). Nonetheless, vestibular (and other body based) cues clearly serve to enhance the fidelity of these representations (Klatzky et al., 1998). In this way, modality independent navigational representations can operate largely in the absence of vestibular cues, although such representations are more flexible and enriched in the presence of modalitydependent forms of representations involving vestibular and other body-based cues (Wolbers et al., 2011).

Another issue to consider is passive vs. active engagement with the environment. Past behavioral studies have provided mixed results regarding whether active navigation results in better performance on spatial tasks like map-drawing and shortcuttaking (Chrastil and Warren, 2012). Neurally, active engagement with stimuli alters neural codes in brain areas important to navigation and memory like the hippocampus (Voss et al., 2011). Even here, though, hippocampal mechanisms like pattern completion/separation (Stokes et al., 2015) still operate under conditions of passive navigation. It remains to be determined, then, the extent to which active vs. passive navigation significantly alters navigation-related neural coding in humans. Furthermore, while we have described how encoding modality might affect spatial representations more generally, exactly how it might differentially affect egocentric vs. allocentric representations both neurally and cognitively remains largely untested. We hope, however, that our discussions above, and Figures 1, 2, will provide some possible theoretical predictions for testing these issues in the future.

\section{CONCLUSION}

To simplify the complexity of our considerations in cognitive neuroscience, it is often helpful to reduce the cognitive processes under consideration to more "elemental" ones, such as the frequently employed dichotomy in spatial navigation between egocentric vs. allocentric spatial information. As discussed above, however, we lack process pure measures of these codes, and must rely on tasks that are more likely to require one reference frame or the other for the optimal solution. To address this issue, we propose the RVD task that we believe utilizes more allocentric information than the commonly used JRD task. We then consider how encoding modality might influence egocentric and allocentric codes, and in particular, how we translate between them. We conclude that while studying human spatial navigation with ongoing neural recordings requires some compromise based on using fMRI/scalp EEG and desktop VR, with some expected changes in how these representations manifest, overall, these will not dramatically alter human navigation codes. Together, we hope the discussion provided here can provide useful 
considerations for research paradigms involving evaluating how spatial information will be acquired and deployed during encoding and retrieval from different modalities, such as desktop VR vs. real-world navigation.

\section{AUTHOR CONTRIBUTIONS}

MS developed these perspectives under the supervision of AE. MS drafted the manuscript and AE provided critical revisions.

\section{REFERENCES}

Aghajan, Z. M., Schuette, P., Fields, T. A., Tran, M. E., Siddiqui, S. M., Hasulak, N. R., et al. (2017). Theta oscillations in the human medial temporal lobe during real-world ambulatory movement. Curr. Biol. 27, 3743-3751.e3. doi: 10.1016/j.cub.2017.10.062

Basri, R., Costa, L., Geiger, D., and Jacobs, D. (1998). Determining the similarity of deformable shapes. Vis. Res. 38, 2365-2385. doi: 10.1016/S0042-6989(98) 00043-1

Bohbot, V. D., Copara, M. S., Gotman, J., and Ekstrom, A. D. (2017). Lowfrequency theta oscillations in the human hippocampus during real-world and virtual navigation. Nat. Commun. 8:14415. doi: 10.1038/ncomms14415

Brunye, T. T., Gagnon, S. A., Waller, D., Hodgson, E., Tower-Richardi, S., and Taylor, H. A. (2012). Up north and down south: implicit associations between topography and cardinal direction. Q. J. Exp. Psychol. 65, 1880-1894. doi: 10. 1080/17470218.2012.663393

Burgess, N. (2006). Spatial memory: how egocentric and allocentric combine. Trends Cogn. Sci. 10, 551-557. doi: 10.1016/j.tics.2006.10.005

Byrne, P., Becker, S., and Burgess, N. (2007). Remembering the past and imagining the future: a neural model of spatial memory and imagery. Psychol. Rev. 114, 340-375. doi: 10.1037/0033-295X.114.2.340

Chance, S. S., Gaunet, F., Beall, A. C., and Loomis, J. M. (1998). Locomotion mode affects the updating of objects encountered during travel: the contribution of vestibular and proprioceptive inputs to path integration. Presence 7, 168-178. doi: 10.1162/105474698565659

Chrastil, E. R., and Warren, W. H. (2012). Active and passive contributions to spatial learning. Psychon. Bull. Rev. 19, 1-23. doi: 10.3758/s13423-011-0182-x

Chrastil, E. R., and Warren, W. H. (2013). Active and passive spatial learning in human navigation: acquisition of survey knowledge. J. Exp. Psychol. Learn. Mem. Cogn. 39, 1520-1537. doi: 10.1037/a0032382

Eichenbaum, H. (2017). The role of the hippocampus in navigation is memory. J. Neurophysiol. 117, 1785-1796. doi: 10.1152/jn.00005.2017

Ekstrom, A., Viskontas, I., Kahana, M., Jacobs, J., Upchurch, K., Bookheimer, S., et al. (2007). Contrasting roles of neural firing rate and local field potentials in human memory. Hippocampus 17, 606-617. doi: 10.1002/hipo.20300

Ekstrom, A. D. (2010). "Navigation in virtual space: psychological and neural aspects," in Encyclopedia of Behavioral Neuroscience, eds G. F. Koob, M. L. Moal, and R. F. Thompson (London: Academic Press), 286-293. doi: 10.1016/B9780-08-045396-5.00171-8

Ekstrom, A. D. (2015). Why vision is important to how we navigate. Hippocampus 25, 731-735. doi: 10.1002/hipo.22449

Ekstrom, A. D., Arnold, A. E., and Iaria, G. (2014). A critical review of the allocentric spatial representation and its neural underpinnings: toward a network-based perspective. Front. Hum. Neurosci. 8:803. doi: 10.3389/fnhum. 2014.00803

Ekstrom, A. D., Huffman, D. J., and Starrett, M. (2017). Interacting networks of brain regions underlie human spatial navigation: a review and novel synthesis of the literature. J. Neurophysiol. 118, 3328-3344. doi: 10.1152/jn.00531.2017

Ekstrom, A. D., and Isham, E. A. (2017). Human spatial navigation: representations across dimensions and scales. Curr. Opin. Behav. Sci. 17, 84-89. doi: 10.1016/j. cobeha.2017.06.005

Epstein, R. A. (2008). Parahippocampal and retrosplenial contributions to human spatial navigation. Trends Cogn. Sci. 12, 388-396. doi: 10.1016/j.tics.2008. 07.004

\section{FUNDING}

This work was supported by NSF BCS-1630296 (AE).

\section{ACKNOWLEDGMENTS}

The authors would like to thank Derek Huffman, Jared Stokes, and Branden Kolarik for helpful discussions contributing to the development of these ideas.

Gramann, K., Onton, J., Riccobon, D., Mueller, H. J., Bardins, S., and Makeig, S. (2010). Human brain dynamics accompanying use of egocentric and allocentric reference frames during navigation. J. Cogn. Neurosci. 22, 2836-2849. doi: 10. 1162/jocn.2009.21369

Han, X., and Becker, S. (2014). One spatial map or many? Spatial coding of connected environments. J. Exp. Psychol. Learn. Mem. Cogn. 40, 511-531. doi: $10.1037 / \mathrm{a} 0035259$

He, Q., Mcnamara, T. P., and Kelly, J. W. (2017). "Environmental and idiothetic cues to reference frame selection in path integration," in Spatial Cognition X, eds T. Barkowsky, H. Burte, C. Hölscher, and H. Schultheis (Cham: Springer International Publishing), 137-156.

Holmes, M. C., and Sholl, M. J. (2005). Allocentric coding of object-to-object relations in overlearned and novel environments. J. Exp. Psychol. Learn. Mem. Cogn. 31, 1069-1087. doi: 10.1037/0278-7393.31.5.1069

Jacobs, J., Kahana, M. J., Ekstrom, A. D., Mollison, M. V., and Fried, I. (2010). A sense of direction in human entorhinal cortex. Proc. Natl. Acad. Sci. U.S.A. 107, 6487-6492. doi: 10.1073/pnas.0911213107

Kelly, J. W., Avraamides, M. N., and Loomis, J. M. (2007). Sensorimotor alignment effects in the learning environment and in novel environments. J. Exp. Psychol. Learn. Mem. Cogn. 33, 1092-1107. doi: 10.1037/0278-7393.33.6.1092

Kelly, J. W., and McNamara, T. P. (2009). Facilitated pointing to remembered objects in front: evidence for egocentric retrieval or for spatial priming? Psychon. Bull. Rev. 16, 295-300. doi: 10.3758/PBR.16.2.295

Klatzky, R. L., Loomis, J. M., Beall, A. C., Chance, S. S., and Golledge, R. G. (1998). Spatial updating of self-position and orientation during real, imagined, and virtual locomotion. Psychol. Sci. 9, 293-298. doi: 10.1111/1467-9280.00058

Kozak, J. J., Hancock, P. A., Arthur, E. J., and Chrysler, S. T. (1993). Transfer of training from virtual-reality. Ergonomics 36, 777-784. doi: 10.1080/ 00140139308967941

Miller, J. F., Neufang, M., Solway, A., Brandt, A., Trippel, M., Mader, I., et al. (2013). Neural activity in human hippocampal formation reveals the spatial context of retrieved memories. Science 342, 1111-1114. doi: 10.1126/science.1244056

Morris, R. G., Hagan, J. J., and Rawlins, J. N. (1986). Allocentric spatial learning by hippocampectomised rats: a further test of the "spatial mapping" and "working memory" theories of hippocampal function. Q. J. Exp. Psychol. B 38, 365-395.

Mou, W., and McNamara, T. P. (2002). Intrinsic frames of reference in spatial memory. J. Exp. Psychol. Learn. Mem. Cogn. 28, 162-170. doi: 10.1037/02787393.28.1.162

Mou, W., Mcnamara, T. P., Rump, B., and Xiao, C. (2006). Roles of egocentric and allocentric spatial representations in locomotion and reorientation. J. Exp. Psychol. Learn. Mem. Cogn. 32, 1274-1290. doi: 10.1037/0278-7393.32.6. 1274

Mou, W., Mcnamara, T. P., Valiquette, C. M., and Rump, B. (2004). Allocentric and egocentric updating of spatial memories. J. Exp. Psychol. Learn. Mem. Cogn. 30, 142-157. doi: 10.1037/0278-7393.30.1.142

Newman, E. L., Caplan, J. B., Kirschen, M. P., Korolev, I. O., Sekuler, R., and Kahana, M. J. (2007). Learning your way around town: how virtual taxicab drivers learn to use both layout and landmark information. Cognition 104, 231-253. doi: 10.1016/j.cognition.2006.05.013

Paris, R., Joshi, M., He, Q., Narasimham, G., Mcnamara, T. P., and Bodenheimer, B. (2017). "Acquisition of survey knowledge using walking in place and resetting methods in immersive virtual environments," in Proceedings of the ACM Symposium on Applied Perception, (Cottbus: ACM). doi: 10.1145/3119881. 3119889 
Richardson, A. E., Montello, D. R., and Hegarty, M. (1999). Spatial knowledge acquisition from maps and from navigation in real and virtual environments. Mem. Cogn. 27, 741-750. doi: 10.3758/BF03211566

Ruddle, R. A., and Lessels, S. (2006). For efficient navigational search, humans require full physical movement, but not a rich visual scene. Psychol. Sci. 17, 460-465. doi: 10.1111/j.1467-9280.2006.01728.x

Ruddle, R. A., and Lessels, S. (2009). The benefits of using a walking interface to navigate virtual environments. ACM Trans. Comput. Hum. Interact. 16:5. doi: 10.1145/1502800.1502805

Ruddle, R. A., Volkova, E., Heinrich, H., and Lthoff, B. (2011). Walking improves your cognitive map in environments that are large-scale and large in extent. ACM Trans. Comput. Hum. Interact. 18, 1-20. doi: 10.1145/1970378.1970384

Russell, N. A., Horii, A., Smith, P. F., Darlington, C. L., and Bilkey, D. K. (2006). Lesions of the vestibular system disrupt hippocampal theta rhythm in the rat. J. Neurophysiol. 96, 4-14. doi: 10.1152/jn.00953.2005

Sederberg, P. B., Kahana, M. J., Howard, M. W., Donner, E. J., and Madsen, J. R. (2003). Theta and gamma oscillations during encoding predict subsequent recall. J. Neurosci. 23, 10809-10814. doi: 10.1523/JNEUROSCI.23-34-10809. 2003

Shelton, A. L., and McNamara, T. P. (2001). Systems of spatial reference in human memory. Cogn. Psychol. 43, 274-310. doi: 10.1006/cogp.2001.0758

Sholl, M. J. (1999). Egocentric frames of reference used for the retrieval of survey knowledge learned by map and navigation. Spat. Cogn. Comput. 1, 475-494. doi: 10.1023/A:1010047911951

Snow, J. C., Skiba, R. M., Coleman, T. L., and Berryhill, M. E. (2014). Real-world objects are more memorable than photographs of objects. Front. Hum. Neurosci. 8:837. doi: 10.3389/fnhum.2014.00837

Starrett, M. J., Stokes, J. D., Huffman, D. J., Ferrer, E., and Ekstrom, A. D. (in press). Learning-dependent evolution of spatial representations in large-scale virtual environments. J. Exp. Psychol. Learn. Mem. Cogn.

Stokes, J., Kyle, C., and Ekstrom, A. D. (2015). Complementary roles of human hippocampal subfields in differentiation and integration of spatial context. J. Cogn. Neurosci. 27, 546-559. doi: 10.1162/jocn_a_00736

Taube, J. S., Valerio, S., and Yoder, R. M. (2013). Is navigation in virtual reality with FMRI really navigation? J. Cogn. Neurosci. 25, 1008-1019. doi: 10.1162/jocn_a_ 00386

Trullier, O., Wiener, S. I., Berthoz, A., and Meyer, J. A. (1997). Biologically based artificial navigation systems: review and prospects. Prog. Neurobiol. 51, 483-544. doi: 10.1016/S0301-0082(96)00060-3

Tulving, E., and Thomson, D. M. (1973). Encoding specificity and retrieval processes in episodic memory. Psychol. Rev. 80, 352-373. doi: 10.1037/h002 0071

Voss, J. L., Gonsalves, B. D., Federmeier, K. D., Tranel, D., and Cohen, N. J. (2011). Hippocampal brain-network coordination during volitional exploratory behavior enhances learning. Nat. Neurosci. 14, 115-120. doi: 10.1038/nn.2693
Waller, D., and Hodgson, E. (2006). Transient and enduring spatial representations under disorientation and self-rotation. J. Exp. Psychol. Learn. Mem. Cogn. 32, 867-882. doi: 10.1037/0278-7393.32.4.867

Waller, D., and Hodgson, E. (2013). "Sensory contributions to spatial knowledge of real and virtual environments," in Human Walking in Virtual Environments: Perception, Technology, and Applications, eds F. Steinicke, Y. Visell, J. Campos, and A. Lécuyer (New York, NY: Springer), 3-26. doi: 10.1007/978-1-44198432-6_1

Wang, R. F. (2017). Spatial updating and common misinterpretations of spatial reference frames. Spat. Cogn. Comput. 77, 222-249. doi: 10.1080/13875868. 2017.1304394

Wang, R. F., and Spelke, E. S. (2000). Updating egocentric representations in human navigation. Cognition 77, 215-250. doi: 10.1016/S0010-0277(00)00 105-0

Watrous, A. J., Fried, I., and Ekstrom, A. D. (2011). Behavioral correlates of human hippocampal delta and theta oscillations during navigation. J. Neurophysiol. 105, 1747-1755. doi: 10.1152/jn.00921.2010

Watrous, A. J., Tandon, N., Conner, C. R., Pieters, T., and Ekstrom, A. D. (2013). Frequency-specific network connectivity increases underlie accurate spatiotemporal memory retrieval. Nat. Neurosci. 16, 349-356. doi: 10.1038/nn. 3315

Wolbers, T., Klatzky, R. L., Loomis, J. M., Wutte, M. G., and Giudice, N. A. (2011). Modality-independent coding of spatial layout in the human brain. Curr. Biol. 21, 984-989. doi: 10.1016/j.cub.2011.04.038

Yaffe, R. B., Kerr, M. S., Damera, S., Sarma, S. V., Inati, S. K., and Zaghloul, K. A. (2014). Reinstatement of distributed cortical oscillations occurs with precise spatiotemporal dynamics during successful memory retrieval. Proc. Natl. Acad. Sci. U.S.A. 111, 18727-18732. doi: 10.1073/pnas.1417017112

Yassa, M. A. (2018). Brain rhythms: higher-frequency theta oscillations make sense in moving humans. Curr. Biol. 28, R70-R72. doi: 10.1016/j.cub.2017.11.045

Zhang, H., Zherdeva, K., and Ekstrom, A. D. (2014). Different "routes" to a cognitive map: dissociable forms of spatial knowledge derived from route and cartographic map learning. Mem. Cogn. 42, 1106-1117. doi: 10.3758/s13421014-0418-x

Conflict of Interest Statement: The authors declare that the research was conducted in the absence of any commercial or financial relationships that could be construed as a potential conflict of interest.

Copyright (C) 2018 Starrett and Ekstrom. This is an open-access article distributed under the terms of the Creative Commons Attribution License (CC BY). The use, distribution or reproduction in other forums is permitted, provided the original author(s) and the copyright owner(s) are credited and that the original publication in this journal is cited, in accordance with accepted academic practice. No use, distribution or reproduction is permitted which does not comply with these terms. 